\title{
Breathlessness, physical activity and sustainability of healthcare
}

To the Editor:

We welcome the important paper by GRøNSETH et al. [1], which used data from the Burden of Obstructive Lung Disease (BOLD) project to highlight the prevalence of breathlessness across the world. As well as being significant because it is a source of suffering that should be ameliorated, we believe that attention to breathlessness, particularly in midlife, has important implications for sustainable healthcare because it allows timely diagnosis, would drive the promotion of physical activity, forms part of a strategy to reduce the burden of comorbidities and would have beneficial environmental effects.

Ensuring that health systems remain sustainable requires the prevention of future ill health [2]. Breathlessness in an individual may or may not indicate the presence of disease, but if it triggers a timely diagnosis of chronic obstructive pulmonary disease (COPD) or other conditions it offers the opportunity to intervene at an early stage with evidence-based therapies including, in COPD, intensive smoking cessation support, influenza vaccination and pulmonary rehabilitation [3]. Late presentation is a major concern in COPD, with many patients not diagnosed until they are admitted to hospital with an acute exacerbation [4].

As the authors note, the data they collected could only account for $13 \%$ of the variance in dyspnoea [1]. Other lung function parameters, such as transfer factor, have been shown to wield more prognostic power in COPD than spirometry [5] but, even in an extensively characterised population of COPD patients, lung function and other clinical parameters could explain only 32\% of the variance in Medical Research Council score, with transfer factor of the lung for carbon monoxide, residual volume/total lung capacity ratio and exacerbation rate retained in a stepwise regression model [6]. Lung health screening in primary care confirms that breathlessness is common and associated both with reduced self-reported physical activity and reduced quadriceps strength, indicating the presence of deconditioning in healthy individuals as well as those with abnormal spirometry [7]. Increase in physical activity in midlife is associated with improved survival [8] and support to increase physical activity should be part of a structured approach to the management of breathlessness, and given as much importance as the medical management of specific pathologies. An increase in active transport (walking or cycling) is a key tool for promoting fitness and particularly desirable for respiratory health, as increased physical activity may reduce lung function decline [9], while simultaneously reducing the financial and carbon/climate change cost to the environment of transport generally, as well as particulate emissions from car exhausts, which have specific noxious effects on lung health.

Finally, the need for concerted efforts to increase physical activity was highlighted in a recent UK Dept of Health Framework document for the management of comorbidities [10]. Many patients with COPD have comorbidities, including cardiovascular disease, diabetes, osteoporosis and anxiety, all of which may, to an extent, be prevented or reversed by the promotion of physical activity in breathless individuals who have, or may otherwise go on to develop, long-term conditions.

Attention to breathlessness in midlife has the potential to significantly improve the identification of early disease and prevent the development of health problems, and would promote the sustainability at a social, financial and environmental level that is needed to improve patient care today and enable systems to deliver high-quality healthcare tomorrow.

@ERSpublications

Targeting breathlessness is important for delivering sustainable future healthcare http://ow.ly/AgKoA

Amina Aitsi-Selmi ${ }^{1}$ and Nicholas S. Hopkinson ${ }^{2}$

${ }^{1}$ Institute of Health Equity, Dept of Epidemiology and Public Health, London, UK. ${ }^{2}$ NIHR Respiratory Biomedical Research Unit at Royal Brompton and Harefield NHS Foundation Trust and Imperial College London, London, UK.

Correspondence: Nicholas S. Hopkinson, Royal Brompton Hospital, Fulham Rd, London, SW3 6NP, UK.

E-mail: n.hopkinson@ic.ac.uk

Received: June 202014 | Accepted: June 222014

Conflict of interest: None declared. 


\section{References}

1 Grønseth R, Vollmer WM, Hardie JA, et al. Predictors of dyspnoea prevalence: results from the BOLD study. Eur Respir J 2014; 43: 1610-1620.

2 NHS Sustainable Development Unit. Sustainability in the NHS: Health Check 2012. Cambridge, NHS Sustainable Development Unit, 2012. Available from: www.sduhealth.org.uk/documents/publications/Sustainability_in_the_ NHS_Health_Check_2012_On-Screen_Version.pdf

3 British Thoracic Society, Primary Care Respiratory Society UK. IMPRESS Guide to the Relative Value of COPD Interventions. British Thoracic Society Reports, Vol. 4, Issue 2, 2012. Available from: www.impressresp.com/index. php?option $=$ com_docman\&task $=$ doc_view\&gid $=52 \& I t e m i d=82$

4 Bastin AJ, Starling L, Ahmed R, et al. High prevalence of undiagnosed and severe chronic obstructive pulmonary disease at first hospital admission with acute exacerbation. Chron Respir Dis 2010; 7: 91-97.

5 Boutou AK, Shrikrishna D, Tanner RJ, et al. Lung function indices for predicting mortality in COPD. Eur Respir J 2013; 42: 616-625.

6 Kelly JL, Bamsey O, Smith C, et al. Health status assessment in routine clinical practice: the chronic obstructive pulmonary disease assessment test score in outpatients. Respiration 2012; 84: 193-199.

$7 \quad$ Kelly JL, Elkin SL, Fluxman J, et al. Breathlessness and skeletal muscle weakness in patients undergoing lung health screening in primary care. COPD 2013; 10: 40-54.

8 Byberg L, Melhus $\mathrm{H}$, Gedeborg R, et al. Total mortality after changes in leisure time physical activity in 50 year old men: 35 year follow-up of population based cohort. BMJ 2009; 338: b688.

9 Hopkinson NS, Polkey MI. Does physical inactivity cause chronic obstructive pulmonary disease? Clin Sci 2010 118: $565-572$.

10 Dept of Health. Comorbidities: a Framework of Principles for System-wide Action. London, Dept of Health, 2014 Available from: www.gov.uk/government/uploads/system/uploads/attachment_data/file/307143/Comorbidities_ framework.pdf

\section{Low minimal inhibitory concentrations of linezolid against multidrug-resistant tuberculosis strains}

To the Editor:

We are following the debate about the administration and the preferred dosage of linezolid in the treatment of multidrug-resistant (MDR) tuberculosis (TB) and extensively drug-resistant (XDR) TB with great interest. Linezolid is a high potent drug against M. tuberculosis, but its widespread use is limited due to severe side-effects in long-term treatment, which often occurs after the usage of $600 \mathrm{mg}$ twice a day in bacterial disease for $>28$ days and mainly includes severe haematotoxic effects (i.e. myelosuppression, anaemia, thrombocytopaenia) or polyneuropathy [1]. However, the current standards in MDR- and XDR-TB require several months of treatment. In addition, the administration of $1200 \mathrm{mg}$ linezolid per day leads to high treatment costs, which limits the usage, especially in low-income countries $[1,2]$. Lowering the dosage of linezolid could, therefore, be an effective step towards reducing costs and severe side-effects.

In a letter from Sotgiu et al. [3], published in the European Respiratory Journal, the authors analysed a subgroup of patients enrolled in their previous meta-analysis about the safety, efficacy and tolerability of linezolid in the treatment of MDR-TB [1]. They found, in a crude statistical comparison of their results to the findings by LEE et al. [4] who had provided prospective experimental data for the safety of linezolid in the treatment of XDR-TB, that the best risk-benefit profile was a daily dose of $\leqslant 600 \mathrm{mg}$ [3]. This suggestion was supported by three other studies, which showed some evidence that lower, but yet effective, dosage of linezolid may reduce both toxicity and cost $[2,5,6]$. In this context, drug susceptibility testing is necessary to determine efficacy of linezolid against a wide range of $M$. tuberculosis strains and could provide a further rationale for the application of a lower dosage.

For this purpose we retrospectively evaluated the in vitro susceptibility test results of linezolid from clinical routine assessment via minimal inhibitory concentrations (MICs) against $148 \mathrm{M}$. tuberculosis strains including 18 MDR-TB strains isolated from patients treated in our centre from 2002 to 2012. Testing for MIC was performed on solid Middlebrook-7H10 agar plates as described elsewhere [7]. MICs were defined 